\section{Perfil das intoxicações na infância atendidas pelo Centro de Informação Toxicológica do Rio Grande do Sul (CIT/RS), Brasil}

\author{
Caseload of poisoning among children treated \\ by the Rio Grande do Sul State Toxicology \\ Information Center (CIT/RS), Brazil
}

\footnotetext{
1 Centro de Informação

Toxicológica do Rio Grande

do Sul, Secretaria

Estadual da Saúde,

Porto Alegre, Brasil.

2 Fundação Faculdade

Federal de Ciências

Médicas de Porto Alegre,

Porto Alegre, Brasil.

3 Serviço de Doenças

Infecciosas e Parasitárias,

Santa Casa de Porto Alegre,

Porto Alegre, Brasil.

${ }^{4}$ Curso de Pós-graduação

em Saúde Coletiva,

Universidade Luterana

do Brasil, Canoas, Brasil.

Correspondência

C. L. J. Ramos

Centro de Informação

Toxicológica do Rio Grande

do Sul, Fundação Estadual

de Produção e Pesquisa

em Saúde, Secretaria

Estadual da Saúde.

Rua Marcelo Gama 1356,

apto. 401, Porto Alegre, RS

90540-041, Brasil

carlaram@terra.com.br
}

\begin{abstract}
This was an analytical, descriptive, cross-sectional study to establish the profile of poisonings among children 0 to 4 years of age reported to the Rio Grande do Sul State Toxicology Information Center (CIT/RS) in Porto Alegre, Brazil, 2003. A total of 1,311 cases were selected and data were extracted on children who had suffered individual accidental poisoning inside the home. From a systematic random sample of 286 patients, 21 interviews were held with the children's parents or guardians, using a structured instrument. The typical profile of poisoning featured: $\leq 1$ year age bracket; male gender; oral exposure; poisonous agent located on living room or bedroom floor; furniture height $<30 \mathrm{~cm}$; time of poisoning from 18:01 to 22:00; time between poisoning and search for emergency medical care less than 30 minutes; analgesics as poisonous agent; medical assessment of purported poisoning as non-toxic, evolving to cure. The data indicate relevant characteristics as to the time of poisoning and place where poisonous agents were located. Definition of a profile helps promote and expand childhood poisoning prevention campaigns by public health agencies.
\end{abstract}

Poisoning; Child Welfare; Accident Prevention
Carla Luiza Job Ramos 1

Maria Beatriz Mostardeiro Targa 2,3

Airton Tetelbom Stein 2,4

\section{Introdução}

As intoxicações não intencionais, principalmente por medicamentos, representam um problema de saúde pública em virtude da elevada prevalência. De acordo com os dados da rede do Sistema Nacional de Informações TóxicoFarmacológicas (SINITOX), da Fundação Oswaldo Cruz (FIOCRUZ), no ano de 2000 ocorreram 8.904 notificações não intencionais por medicamentos 1. Crianças, principalmente menores de cinco anos de idade, por natureza, são mais curiosas. Por estarem na fase oral, o relacionamento com ambiente se dá por meio da prática de levar objetos e substâncias à boca. Além disso, é muito comum que pais e responsáveis pela guarda subestimem seus atos 2,3. Diferenças entre crianças e adultos, no que se refere à susceptibilidade a intoxicações, podem resultar da combinação da toxicocinética, da toxicodinâmica e de fatores de exposição. Os fatores cinéticos são especialmente importantes no período pós-natal, em grande parte como resultado da imaturidade do sistema excretor, seja pelas reduzidas metabolização enzimática e/ou excreção renal. Tal vulnerabilidade específica pode prevalecer durante muito tempo, acompanhando o processo de desenvolvimento e de maturação dos órgãos 4 .

Rahde et al. 5 relataram que crianças entre dois e três anos apresentaram maior freqüência de intoxicações, com 38,6\% de 9.095 intoxi- 
cações notificadas ao Centro de Informação Toxicológica do Rio Grande do Sul (CIT/RS) no período de 1980 a 1986. Nesse estudo, as intoxicações de caráter não intencional representaram 95,2\%, sendo "medicamentos" o agente causal mais comum em 3.637 casos $(40,0 \%)$. Segundo o Relatório de Atendimento 2002 6, em seu banco de dados, o CIT/RS registrou 1.311 (126/10 mil crianças) casos de intoxicação em crianças da faixa etária de 0 a 4 anos de idade no Município de Porto Alegre (31,9\% de 4.103 casos registrados na mesma faixa etária, oriundos de toda a Região Sul do Brasil).

Diante dessas considerações, o estudo tem como objetivo a identificação do perfil das intoxicações em menores de 0 a 4 anos de idade do Município de Porto Alegre, notificadas no CIT/RS no ano de 2003. Visa ainda identificar fatores de risco para a ocorrência de intoxicação.

\section{Material e métodos}

O estudo foi conduzido no banco de dados do CIT/RS, que é um departamento ligado à Fundação Estadual de Produção e Pesquisa em Saúde (FEPPS). A missão do CIT é prestar atendimento, via telefone, à população em geral ou aos profissionais da saúde em face de situações que envolvam acidentes tóxicos. Funciona em regime de plantão permanente nas 24 horas do dia, incluindo os finais de semana e feriados, e conta com uma equipe de técnicos e plantonistas da área da saúde para o atendimento. Comunica-se via linhas telefônicas privativas com hospitais do Município de Porto Alegre e dispõe de uma linha de Discagem Direta Gratuita (DDG) que atende a população em geral. A cada atendimento é preenchida uma Ficha de Notificação e Atendimento pelo profissional que o realiza, a qual, após ser codificada, é incluída e processada em banco de dados estatístico próprio.

Realizou-se um estudo transversal com componentes descritivos e analíticos. Foram utilizados 4.103 registros de casos no banco de dados do CIT/RS, com as informações provenientes de demanda espontânea.

Foram selecionados 1.311 registros de casos de intoxicação individual em crianças com idades entre 0 e 4 anos ocorridos em seus domicílios do Município de Porto Alegre, no período de 1o de janeiro a 31 de agosto de 2003 . Com base nessa lista, foi realizada uma amostra aleatória sistemática de 286 pacientes, con- siderando uma freqüência esperada de armazenamento inadequado de $75,0 \%$ e um erro aceitável de $5 \%$, para um intervalo de confiança de $95 \%$. A amostra foi realizada por meio da seleção de 1 a cada 5 pacientes (um pulo de cinco). Houve limitações no presente estudo, como o difícil acesso às residências e a recusa em participar, característicos de um estudo com base populacional. Foram obtidas 21 entrevistas com os responsáveis legais da criança com intoxicação, tendo sido aplicado um consentimento livre para realização delas. Para coleta de dados, utilizou-se um instrumento estruturado de coleta de dados (ficha investigatória). As variáveis analisadas foram idade, sexo, telefone, endereço, origem (domicílio, clínica de saúde, unidades de saúde públicas, hospitais e outros), avaliação da intoxicação e evolução desta. Analisaram-se, também, as circunstâncias da intoxicação: responsável pela criança no momento do evento (pais, avós, irmãos, doméstica, outro); local do acidente (sala, quarto, cozinha, banheiro, área de serviço, jardim, outro); horário do acidente (8h-10h, 10h01-12h, 12h01-15h, 15h01-18h, 18h01-22h, 22h01-7h59); armazenamento do agente tóxico (armário, gaveta, caixa, bolsa, sobre a mesa, outro); altura do armazenamento do agente tóxico (abaixo de $50 \mathrm{~cm}, 51 \mathrm{~cm}-100 \mathrm{~cm}, 101 \mathrm{~cm}-150 \mathrm{~cm}$, acima de $150 \mathrm{~cm}$ ); agente tóxico; local onde buscou o primeiro atendimento médico (posto de saúde, hospital, clínica, outro); tempo do acidente e o primeiro atendimento (menos de $30 \mathrm{~min}$, 30min-1h, 1h-2h, 2h-3h, mais de 3h); descontaminação (sim, não); mudança de armazenamento (sim, não) e novo local de armazenamento (descrição). Investigou-se, ainda, se ocorreu contato telefônico com o CIT/RS antes do atendimento médico (sim, não), se havia conhecimento do CIT/RS (sim, não) e quem respondeu à entrevista.

Para a coleta de dados e análise, foram utilizados os pacotes estatísticos Epi Info (6.0) e SPSS. O nível de significância foi estabelecido em 0,05. O teste do qui-quadrado foi aplicado para análise comparativa.

O projeto de pesquisa foi avaliado e aprovado por três comitês de ética: Faculdade de Medicina da Pontifícia Universidade Católica do Rio Grande do Sul, Hospital de Pronto-Socorro Municipal de Porto Alegre e Fundação Estadual de Produção e Pesquisa em Saúde. 


\section{Resultados}

Dos 11.859 casos registrados no CIT/RS no período de 1o de janeiro a 31 de agosto de 2003, 593 foram de intoxicação acidental individual em crianças de 0 a 4 anos de idade, nas residências do Município de Porto Alegre. A maioria das solicitações foi oriunda de hospitais com 252 registros, representando $42,5 \%$ do total de casos. O Hospital de Pronto-Socorro $\mathrm{Mu}$ nicipal de Porto Alegre (HPS) configurou-se como o maior informante entre os hospitais de Porto Alegre, com 90 (35,7\%) casos (Figura 1). As ligações residenciais representaram 34,9\% dos casos, com 207 registros, sendo o segundo maior grupo de solicitantes. Dos 593 registros do CIT/RS, a faixa etária em estudo foi a compreendida entre 0 e 4 anos, e a ocorrência mais comum de acidente foi com um ano de idade (28,5\%), sendo $53,3 \%$ do gênero masculino. A via de exposição mais freqüente foi a oral, representando $88,4 \%$ do total de casos. O horário mais freqüente da intoxicação foi o do período das $18 \mathrm{~h} 01$ às $22 \mathrm{~h}$, tendo decorrido menos de 30 minutos entre a exposição e o primeiro contato com o CIT/RS. A maioria das intoxicações apresentou a avaliação de intoxicação como nãotóxica, e, dos 593 casos, 578 evoluíram para a cura (Tabela 1). Na classificação em relação à distribuição por grupos dos agentes tóxicos, verificou-se que os medicamentos foram os mais freqüentes, com 250 casos, tendo como classificação de maior representatividade os analgésicos. Dentre os domissanitários, verificou-se maior freqüência dos alvejantes (Tabela 2).

$\mathrm{Na}$ Tabela 3, podemos analisar as variáveis referentes às 21 entrevistas. Crianças com um ano de idade representaram a maioria das intoxicações, sendo 52,4\% do gênero masculino. Os medicamentos também apresentaram maior freqüência em $23,8 \%$ das entrevistas, e, em $81,0 \%$ das intoxicações, a via de exposição foi a oral. Verificou-se que, em $90,5 \%$ dos casos, o evento foi o primeiro acidente tóxico da casa, e os responsáveis pelas crianças no momento do acidente, em $76,2 \%$ das entrevistas, eram os pais. O local da residência onde se encontravam os agentes tóxicos foi a sala de estar e o quarto (23,8\% em cada) e o local específico (móvel), o chão em $66,7 \%$ entrevistas. O horário da intoxi-

\section{Figura 1}

Distribuição de intoxicações em crianças de 0 a 4 anos (acidente individual) nos domicílios do Município de Porto Alegre, Rio Grande do Sul, Brasil, com busca de atendimento médico em hospitais registrados no Centro de Informação Toxicológica do Rio Grande do Sul (CIT/RS).

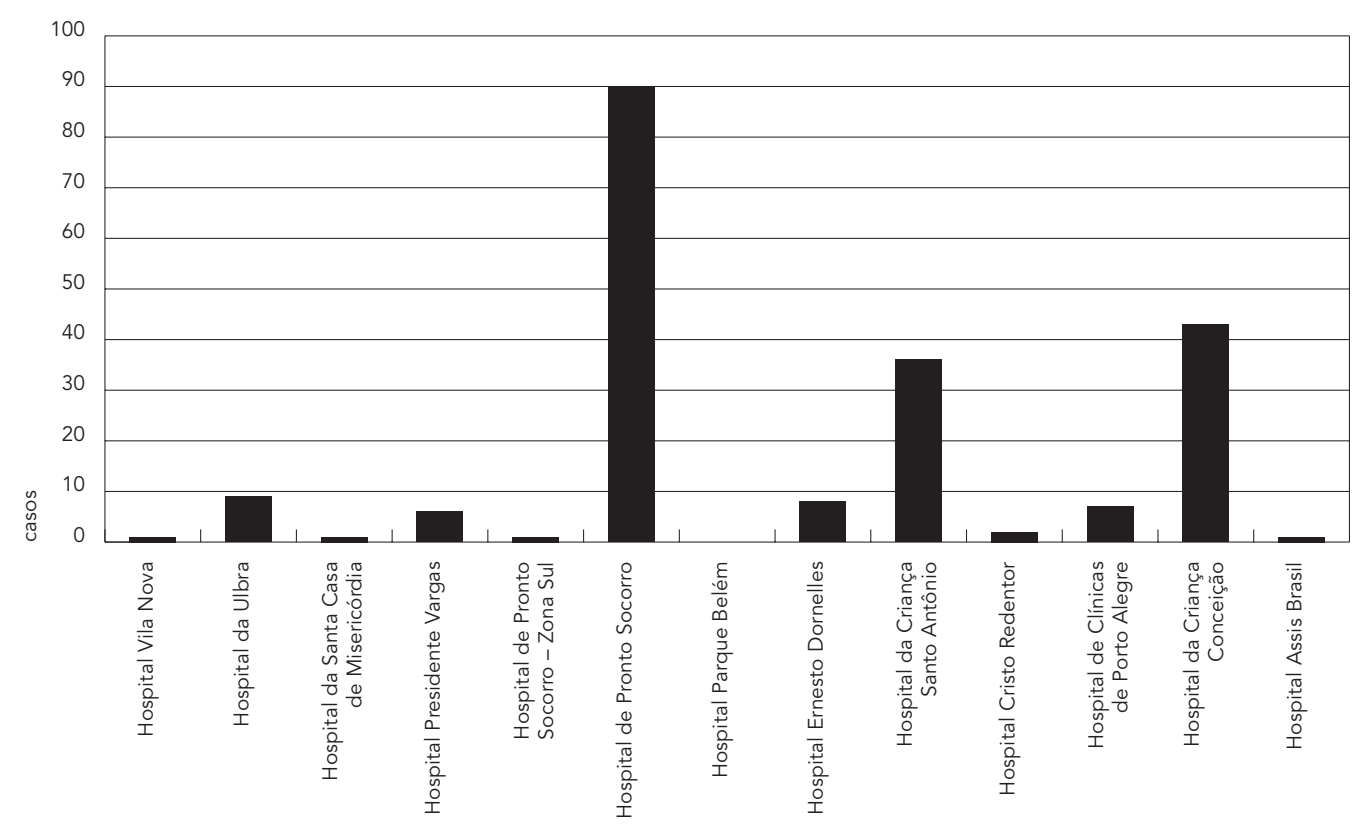


Distribuição das variáveis de intoxicações acidentais individuais em crianças de 0 a 4 anos, de acordo com a origem da solicitação, no Município de Porto Alegre, Rio Grande do Sul, Brasil, 1ㅇ de janeiro a 31 de agosto de 2003.

\begin{tabular}{|c|c|c|c|c|c|c|c|c|c|c|}
\hline & \multicolumn{2}{|c|}{ Clínicas médicas } & \multicolumn{2}{|c|}{ Hospitais } & \multicolumn{2}{|c|}{ Postos de saúde } & \multicolumn{2}{|c|}{ Residências } & \multicolumn{2}{|c|}{ Total } \\
\hline & $n$ & $\%$ & n & $\%$ & n & $\%$ & n & $\%$ & $\mathrm{n}$ & $\%$ \\
\hline \multicolumn{11}{|l|}{ Faixa etária (mediana) } \\
\hline$<1$ ano & 3 & 7,3 & 18 & 7,1 & 4 & 4,3 & 17 & 8,2 & 42 & 7,1 \\
\hline 1 ano & 12 & 29,3 & 63 & 25,0 & 29 & 31,2 & 65 & 31,4 & 169 & 28,5 \\
\hline 2 anos & 11 & 29,3 & 77 & 30,6 & 27 & 29,0 & 38 & 18,4 & 153 & 25,8 \\
\hline 3 anos & 7 & 17,1 & 62 & 24,6 & 21 & 22,6 & 52 & 25,1 & 142 & 23,9 \\
\hline 4 anos & 8 & 19,5 & 32 & 12,7 & 12 & 12,9 & 35 & 16,9 & 87 & 14,7 \\
\hline \multicolumn{11}{|l|}{ Gênero } \\
\hline Masculino & 21 & 51,2 & 135 & 53,6 & 45 & 48,4 & 115 & 55,6 & 316 & 53,3 \\
\hline Feminino & 20 & 48,8 & 117 & 46,4 & 46 & 49,5 & 92 & 44,4 & 275 & 46,4 \\
\hline Não informado & 0 & 0,0 & 0 & 0,0 & 2 & 2,2 & 0 & 0,0 & 2 & 0,3 \\
\hline \multicolumn{11}{|l|}{ Via de exposição } \\
\hline Oral & 39 & 95,1 & 233 & 92,5 & 78 & 83,9 & 174 & 84,1 & 524 & 88,4 \\
\hline \multicolumn{11}{|l|}{ Horário da intoxicação } \\
\hline $8 \mathrm{~h}-10 \mathrm{~h}$ & 2 & 4,9 & 17 & 6,7 & 4 & 4,3 & 12 & 5,8 & 35 & 5,9 \\
\hline $10 h 01-12 h$ & 5 & 12,2 & 36 & 14,3 & 26 & 28,0 & 37 & 17,9 & 104 & 17,5 \\
\hline $12 \mathrm{~h} 01-15 \mathrm{~h}$ & 15 & 36,6 & 47 & 18,7 & 14 & 15,1 & 46 & 22,2 & 122 & 20,6 \\
\hline $15 h 01-18 h$ & 6 & 14,6 & 40 & 15,9 & 15 & 16,1 & 25 & 12,1 & 86 & 14,5 \\
\hline $18 \mathrm{~h} 01-22 \mathrm{~h}$ & 9 & 22,0 & 60 & 23,8 & 20 & 21,5 & 58 & 28,0 & 147 & 24,8 \\
\hline 22h01-07h59 & 3 & 7,3 & 36 & 14,3 & 10 & 10,8 & 19 & 9,2 & 66 & 11,1 \\
\hline Ignorado & 0 & 0,0 & 16 & 6,3 & 4 & 4,3 & 10 & 4,8 & 33 & 5,6 \\
\hline \multicolumn{11}{|l|}{ Tempo até $o$ atendimento } \\
\hline$<30 \mathrm{~min}$ & 14 & 34,1 & 42 & 16,7 & 17 & 18,3 & 125 & 60,4 & 198 & 33,4 \\
\hline $30 \mathrm{~min}-1 \mathrm{~h}$ & 9 & 22,0 & 59 & 23,4 & 25 & 26,9 & 34 & 16,4 & 127 & 21,4 \\
\hline $1 \mathrm{~h}-2 \mathrm{~h}$ & 7 & 17,1 & 39 & 15,5 & 27 & 29,0 & 18 & 8,7 & 91 & 15,3 \\
\hline $2 h-3 h$ & 2 & 4,9 & 29 & 11,5 & 7 & 7,5 & 2 & 1,0 & 40 & 6,7 \\
\hline+ de $3 h$ & 9 & 22,0 & 83 & 32,9 & 17 & 18,3 & 28 & 13,5 & 137 & 23,1 \\
\hline \multicolumn{11}{|l|}{ Agente $t$} \\
\hline Medicamentos & 22 & 53,7 & 122 & 48,4 & 37 & 39,8 & 69 & 33,3 & 250 & 42,2 \\
\hline \multicolumn{11}{|l|}{ Avaliação } \\
\hline Não tóxico & 19 & 46,3 & 62 & 24,6 & 20 & 21,5 & 95 & 45,9 & 196 & 33,1 \\
\hline Provavelmente não tóxico & 10 & 24,4 & 82 & 32,5 & 26 & 28,0 & 66 & 31,9 & 184 & 31,0 \\
\hline Envenenamento não excluído & 2 & 4,9 & 8 & 3,2 & 6 & 6,5 & 5 & 2,4 & 21 & 3,5 \\
\hline Intoxicação leve & 10 & 24,4 & 74 & 29,4 & 33 & 35,5 & 36 & 17,4 & 153 & 25,8 \\
\hline Intoxicação moderada & 0 & 0,0 & 22 & 8,7 & 7 & 7,5 & 5 & 2,4 & 34 & 5,7 \\
\hline Intoxicação grave & 0 & 0,0 & 4 & 1,6 & 1 & 1,1 & 0 & 0,0 & 5 & 0,8 \\
\hline \multicolumn{11}{|l|}{ Evolução } \\
\hline Cura & 40 & 97,6 & 243 & 96,4 & 91 & 97,8 & 204 & 98,6 & 578 & 97,5 \\
\hline Cura com seqüela & 0 & 0,0 & 1 & 0,4 & 0 & 0,0 & 0 & 0,0 & 1 & 0,2 \\
\hline Ignorada & 1 & 2,4 & 8 & 3,2 & 2 & 2,2 & 3 & 1,4 & 14 & 2,4 \\
\hline
\end{tabular}


Distribuição da classificação dos agentes tóxicos em relação aos grupos, considerando-se o total de intoxicações acidentais individuais em crianças de 0 a 4 anos no Município de Porto Alegre, Rio Grande do Sul, Brasil, 1ㅇ de janeiro a 31 de agosto de 2003 .

\begin{tabular}{|c|c|c|c|c|c|}
\hline \multirow[t]{2}{*}{ Grupos } & \multirow[t]{2}{*}{ Classificação } & \multirow[t]{2}{*}{$\mathrm{n}$} & \multirow[t]{2}{*}{$\%$} & \multicolumn{2}{|c|}{ Total } \\
\hline & & & & $\mathrm{n}$ & $\%$ \\
\hline Medicamentos & Analgésicos & 40 & 6,7 & 250 & 42,2 \\
\hline Agrotóxicos de uso agrícola & Pesticidas agrícolas piretróides & 3 & 0,5 & 8 & 1,3 \\
\hline Agrotóxicos de uso doméstico & Pesticidas domésticos piretróides & 21 & 3,5 & 36 & 6,1 \\
\hline Produtos veterinários & Pesticidas veterinários piretróides & 4 & 0,7 & 1 & 0,2 \\
\hline Raticidas & Raticidas & 34 & 5,7 & 35 & 5,9 \\
\hline Domissanitários & Alvejantes & 32 & 5,4 & 93 & 15,7 \\
\hline Cosméticos & Toalete & 24 & 4,0 & 24 & 4,0 \\
\hline Produtos químicos industriais & Derivados de petróleo & 14 & 2,4 & 44 & 7,4 \\
\hline Metais & Outros metais & 9 & 1,5 & 10 & 1,7 \\
\hline Drogas de abuso & Drogas de abuso & 2 & 0,3 & 2 & 0,3 \\
\hline Plantas & Plantas tóxicas & 39 & 6,6 & 46 & 7,8 \\
\hline Alimentos & & 0 & 0,0 & 0 & 0,0 \\
\hline Animais peçonhentos - Serpentes & & 0 & 0,0 & 0 & 0,0 \\
\hline Animais peçonhentos - Aranhas & Aracnídeo não identificado & 9 & 1,5 & 11 & 1,9 \\
\hline Animais peçonhentos - Escorpiões & & 0 & 0,0 & 0 & 0,0 \\
\hline Outros animais peçonhentos & Lagarta urticante/Bicho cabeludo & 2 & 0,3 & 3 & 0,5 \\
\hline Animais não peçonhentos & Insetos/Animais não identificados & 12 & 2,0 & 23 & 3,9 \\
\hline Outros & Outros & 6 & 1,0 & 6 & 1,0 \\
\hline Ignorado & Ignorado & 1 & 0,2 & 1 & 0,2 \\
\hline
\end{tabular}

cação que apresentou maior freqüência foi o período entre 18h01-22h. Não foi necessária a busca por atendimento médico, posteriormente à orientação do plantão do CIT/RS, em 76,0\% dos casos entrevistados, e o tempo entre o acidente tóxico e a consulta foi de menos de 30 minutos em $71,4 \%$ das entrevistas. Em 76,2\% das entrevistas realizadas, ficou claro que os responsáveis realizaram alteração de conduta e/ou mudança de local de acesso aos agentes tóxicos. A avaliação da intoxicação mais freqüente foi a de acidente não tóxico, evoluindo para a cura em $100,0 \%$ dos casos.

Nas entrevistas, verificou-se que foram realizados mais procedimentos de descontaminação em crianças do gênero masculino $(\mathrm{p}=0,044)$ (Figura 2).

\section{Discussão e conclusão}

Os medicamentos têm sido relatados, nas últimas décadas, pelos centros de referência mundiais em intoxicação, como os maiores causadores de agravo na infância, principalmente na faixa etária de 0 a 4 anos $1,7,8,9,10,11,12,13,14,15$. Não há qualquer dúvida quanto à verificação da inexistência de sazonalidade, bem como quanto ao gênero, via de exposição e agente tóxico, na distribuição dos casos de intoxicação ocorridos em crianças com idade entre 0 e 4 anos, considerando-se seja a estatística do CIT/RS, seja o resultado do trabalho 6 .

Soori 7 identificou que $89,0 \%$ das intoxicações com crianças ocorrem dentro de casa, $79,0 \%$ destas pertencem à faixa etária de 2 a 4 anos e que, em $75,0 \%$ dos casos, os produtos tóxicos estavam acessíveis. Nos Estados Unidos, foram relatados mais de 2 milhões de exposições tóxicas em humanos (2.201.156) no ano de 1999, cujas ocorrências foram notificadas por 60 dos 64 centros de controle de intoxicações existentes naquele país. Em 52,0\% desses casos, as vítimas foram crianças menores de seis anos de idade, raramente resultando em mortalidade 8 . Medidas de prevenção de intoxicações em crianças, como a da instituição de Embalagem Especial de Proteção à Criança (EEPC), implementada nos Estados Unidos e Canadá, são eficazes, depois das quais os índices de intoxicação foram reduzidos em até $35,0 \%$ no período de 1969 a 1972 . Mesmo assim, atualmente, a incidência de intoxicações na infância ainda é preocupante. Em nosso país, 
a EEPC ainda não deixou de ser um Projeto de Lei, que vem tramitando há mais de dez anos sem conseguir sua aprovação 16,17,18,19,20.

De acordo com o relatório anual de 2003 da American Association of Poison Control Centers 8 , a avaliação do acidente tóxico apresentou mediana de intoxicação provavelmente não tóxica, conforme as informações obtidas nos dados gerais e nas residências e entrevista.

Soori 7 identificou, em um estudo caso-controle e descrição epidemiológica, que 89,0\% das crianças se intoxicam dentro de casa. Dados similares foram verificados neste trabalho, destacando-se o chão da sala de estar ou o quarto como ambientes da residência em que mais freqüentemente dá-se a intoxicação. Quanto ao horário de ocorrência dos acidentes tóxicos, identificou-se que o período compreendido entre 18h01-22h apresentou maior freqüência, e os responsáveis pelas crianças no momento da intoxicação buscaram atendimento médico em menos de 30 minutos.

Os dados ressaltam que as condutas das campanhas de divulgação para alertar os pais sobre os acidentes tóxicos com crianças estão corretas e indicam que a precocidade do atendimento médico e a busca por informações nos centros de controle e informação são eficazes $16,17,18,19$.

A instituição do Dia Estadual de Prevenção de Acidentes Tóxicos, quando pessoas ligadas a todos os órgãos públicos de saúde se voltam para tratar de assuntos relacionados à diminuição de intoxicações, foi uma medida tomada pela Secretaria Estadual da Saúde do Rio Grande do Sul para a prevenção de intoxicação, principalmente em crianças 16,21. Tal medida foi copiada da Semana Nacional de Prevenção de Acidentes Tóxicos, realizada pelo Congresso norteamericano. Outras providências tomadas atualmente são a distribuição de folhetos informativos contendo tópicos de prevenção de intoxicações no lar, o Projeto Saúde e Educação no Parque, o Projeto Educar, o Projeto Gibi, entre outros, havendo, ainda, necessidade de desenvolver o planejamento de ações preventivas das intoxicações infantis mais efetivas. Para que isso ocorra com embasamento, devem-se identificar as características dos indivíduos que buscam o CIT/RS, visando diminuir os riscos de futuros acidentes 9,10,11,12,13,22,23.

Os procedimentos de descontaminação em seus domicílios, orientados pelo plantão do CIT/RS, provocaram diminuição da busca por entidades de saúde, postos de saúde, clínicas médicas e/ou hospitais, deixando melhor acesso àquelas que realmente necessitavam de procedimentos médico-hospitalares,
Tabela 3

Distribuição das variáveis faixa etária, gênero, via de exposição, horário da intoxicação, tempo decorrido, agente tóxico, avaliação e evolução, coletadas das 21 entrevistas realizadas. Município de Porto Alegre, Rio Grande do Sul, Brasil, 1ㅇ de janeiro a 31 de agosto de 2003.

\begin{tabular}{|c|c|c|}
\hline & \multicolumn{2}{|c|}{ Entrevistas } \\
\hline & $\mathrm{n}$ & $\%$ \\
\hline \multicolumn{3}{|l|}{ Faixa etária (mediana) } \\
\hline$<1$ ano & 0 & 0,0 \\
\hline 1 ano & 9 & 42,9 \\
\hline 2 anos & 4 & 19,0 \\
\hline 3 anos & 5 & 23,8 \\
\hline 4 anos & 3 & 14,3 \\
\hline \multicolumn{3}{|l|}{ Gênero } \\
\hline Masculino & 11 & 52,4 \\
\hline Feminino & 10 & 47,6 \\
\hline Não informado & 0 & 0,0 \\
\hline \multicolumn{3}{|l|}{ Via de exposição } \\
\hline Oral & 17 & 81,0 \\
\hline \multicolumn{3}{|l|}{ Local da residência } \\
\hline Sala de estar & 5 & 23,8 \\
\hline Sala de jantar & 0 & 0,0 \\
\hline Quarto & 5 & 23,8 \\
\hline Cozinha & 2 & 9,5 \\
\hline Banheiro & 2 & 9,5 \\
\hline Área de serviço & 1 & 4,8 \\
\hline Jardim & 4 & 19,0 \\
\hline Outro & 2 & 9,5 \\
\hline \multicolumn{3}{|l|}{ Móvel } \\
\hline Armário & 3 & 14,3 \\
\hline Gaveta & 1 & 4,8 \\
\hline Caixa & 0 & 0,0 \\
\hline Bolsa & 0 & 0,0 \\
\hline Sobre a mesa & 3 & 14,3 \\
\hline Chão & 14 & 66,7 \\
\hline \multicolumn{3}{|l|}{ Horário da intoxicação } \\
\hline 08h-10h & 4 & 19,0 \\
\hline $10 h 01-12 h$ & 5 & 23,8 \\
\hline $12 \mathrm{~h} 01-15 \mathrm{~h}$ & 3 & 14,3 \\
\hline $15 h 01-18 h$ & 2 & 9,5 \\
\hline $18 \mathrm{~h} 01-22 \mathrm{~h}$ & 6 & 28,6 \\
\hline 22h01-07h59 & 1 & 4,8 \\
\hline \multicolumn{3}{|l|}{ Tempo até o atendimento } \\
\hline Menos de 30min & 15 & 71,4 \\
\hline \multicolumn{3}{|l|}{ Agente tóxico } \\
\hline Medicamentos & 5 & 23,8 \\
\hline \multicolumn{3}{|l|}{ Avaliação } \\
\hline Não tóxico & 8 & 38,1 \\
\hline Provavelmente não tóxico & 7 & 33,3 \\
\hline Intoxicação leve & 4 & 19,0 \\
\hline \multicolumn{3}{|l|}{ Evolução } \\
\hline Cura & 21 & 100,0 \\
\hline
\end{tabular}




\section{Figura 2}

Procedimentos de descontaminação conforme sexo, verificados nas entrevistas com pais de crianças entre 0 e 4 anos.

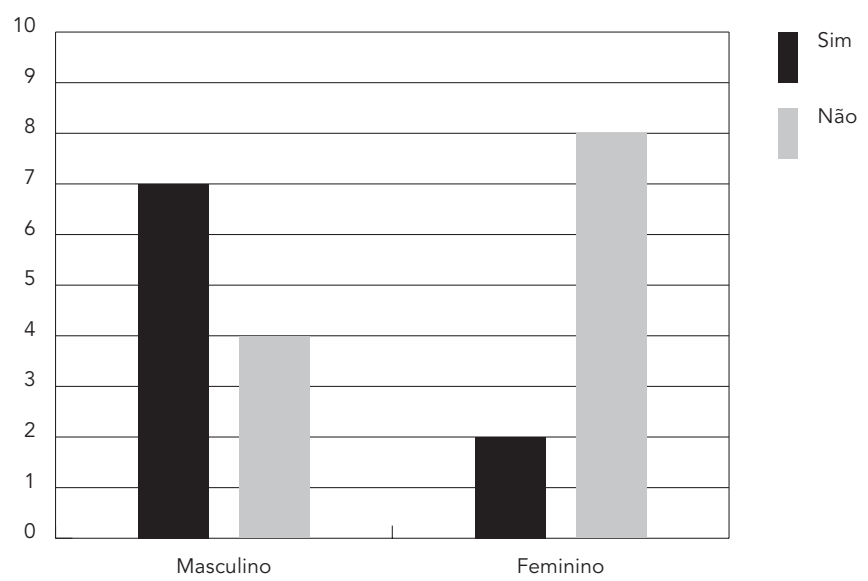

$\chi^{2}=4,073 ; p=0,044$ diminuindo, assim, os custos para os órgãos públicos.

O perfil da intoxicação acidental individual em crianças de 0 a 4 anos de idade no Município de Porto Alegre apresenta as seguintes variáveis: faixa etária de um ano de idade; gênero masculino; via de exposição oral; agente tóxico localizado no chão da sala de estar ou no quarto da residência; altura do móvel inferior a $30 \mathrm{~cm}$; horário da intoxicação entre 18h01-22h; tempo entre o acidente e a busca por uma conduta médica menor que 30 minutos; medicamento analgésico como agente tóxico; avaliação da intoxicação como a não tóxica, caso em que a criança evolui para a cura.

As recomendações feitas com base neste estudo destacam o horário da intoxicação, bem como o local exato em que se encontravam os agentes tóxicos. A definição do perfil possibilita orientar e incrementar as campanhas de prevenção de acidentes tóxicos na infância.

\section{Colaboradores}

C. L. J. Ramos, M. B. M. Targa e A. T. Stein participaram da concepção, análise e interpretação dos dados e da redação, revisão crítica, leitura e aprovação do artigo. 
1. Sistema Nacional de Informações Tóxico-Farmacológicas, Centro de Informação Científica e Tecnológica, Fundação Oswaldo Cruz. Casos registrados de intoxicação humana e envenenamento. Brasil, 2000. http://www.fiocruz.br/sinitox/ (acessado em 26/Out/2004).

2. Gina A, Christine MS. Pediatric toxicologic concerns. Emerg Med Clin North Am 2002; 20:223-47.

3. Lessa CAS. Medicamentos líquidos mais vendidos no Brasil que podem levar a óbito uma criança com o conteúdo de um frasco comercial [Monografia de Especialização]. Porto Alegre: Instituto de Toxicologia, Pontifícia Universidade Católica do Rio Grande do Sul; 2001.

4. Schwenk M, Gundert-Remy U, Heinemeyer G, Olejniczak K, Stahlmann R, Kaufmann W, et al. Children as a sensitive subgroup and their role in regulatory toxicology: DGPT workshop report. Arch Toxicol 2003; 77:2-6.

5. Rahde AF, Wiehe M, Rosa RS. Intoxicações na infância - atendimentos no CIT/RS no período 1980-1986. Revista Científica, Maternidade, Infância e Ginecologia 1987; 7:24-8.

6. Centro de Informação Toxicológica, Secretaria Estadual da Saúde do Rio Grande do Sul. Relatório de atendimento, 2002. Porto Alegre: Centro de Informação Toxicológica, Secretaria Estadual da Saúde do Rio Grande do Sul; 2003.

7. Soori H. Developmental risk factors for unintentional childhood poisoning. Saudi Med J 2001; 22:227-30.

8. Litovitz TL, Klein-Schwartz W, White S, Cobaugh DJ, Youniss J, Drab A, et al. 1999 Annual report of the American Association of Poison Control Centers' toxic exposure surveillance system. Am J Emerg Med 2000; 18:514-7.

9. Ozanne-Smith J, Day L, Parsons B, Tibballs J, Dobbin M. Childhood poisoning: access and prevention. J Paediatr Child Health 2001; 37:262-5.

10. Chein C, Marriott JL, Ashby K, Ozanne-Smith J. Unintentional ingestion of over the counter medications in children less than 5 years old. J Paediatr Child Health 2003; 39:264-9.

11. Shannon M. Primary care: ingestion of toxic substances by children. N Eng J Med 2000; 342:18691.
12. Martin TC, Brinkman W. The spectrum of accidental childhood poisoning in the Caribbean. Rev Panam Salud Publica 2002; 12:313-6.

13. Marchi AG, Renier S, Messi G, Barbone F. Childhood poisoning: a population study in Trieste, Italy, 1975-1994. J Clin Epidemiol 1998; 51:687-95.

14. Trainor JL, Krug SE. The training of pediatric residents in the care of acutely ill and injured children. Arch Pediatr Adolesc Med 2000; 154:1154-9.

15. American Association of Poison Control Centers. 2003 annual report of the American Association of Poison Control Centers Toxic Exposure Surveillance System. Washington DC: American Association of Poison Control Centers; 2004.

16. Schers RG. Prevention of childhood poisoning. Pediatr Clin North Am 1970; 17:713-27.

17. Clarke A, Walton WW. Effect of safety packaging on aspirin ingestion by children. Pediatrics 1979; 63:687-93.

18. Walton WW. An evaluation of the Poison Prevention Packaging Act. Pediatrics 1982; 69:363-70.

19. Rodgers GB. The safety effects of child-resistant packaging for oral prescription drugs. Two decades of experience. JAMA 1996; 275:1661-5.

20. Brasil. Projeto de Lei n. 4.841, de 30 de novembro de 1994. Determina a utilização de Embalagem Especial de Proteção à Criança - EEPC - em medicamentos e produtos químicos de uso doméstico que apresentem potencial de risco à saúde. Brasília: Congresso Nacional; 1994.

21. Rio Grande do Sul. Projeto de Lei n. 11.804, de 2002. Institui o dia 20 de agosto como o "Dia Estadual de Prevenção de Acidentes Tóxicos”. Diário Oficial do Estado do Rio Grande do Sul 2002; 17 jun.

22. O'Donnel J, Brown FD, Beattie TF. Accidental child poisoning: child resistant packaging should be used on all over the counter drugs. BMJ 1998; 316:1460-1.

23. Centro de Informação Toxicológica do Rio Grande do Sul, Secretaria Estadual da Saúde do Rio Grande do Sul. Projeto Educar: módulo I, automedicação e consumo abusivo de medicamentos. Porto Alegre: Centro de Informação Toxicológica do Rio Grande do Sul; 2003.

Recebido em 15/Dez/2004

Versão final reapresentada em 01/Mar/2005

Aprovado em 07/Mar/2005 